SECTION 2. Applied mathematics. Mathematical modeling.

Shevtsov Alexandr Nikolayevich

candidate of technical Sciences,

President of International Academy of Theoretical \& Applied Sciences, Kazakhstan

\title{
ABOUT SOME ALGORITHMS FOR CONSTRUCTING THREE-DIMENSIONAL DYNAMICAL MODELS
}

Abstract: This article presents The algorithms on Delphi, to build a mathematical model of the motion of particles, their interaction with each other and the environment.

Key words: algorithms, model, Delphi.

\section{О НЕКОТОРЫХ АЛГОРИТМАХ ПОСТРОЕНИЯ ТРЕХМЕРНЫХ ДИНАМИЧЕСКИХ МОДЕЛЕЙ}

Аннотация: В данной статье приведень алгоритмы на Дельфи, для построения математической модели движения частии, их взаимодействия друг с другом и внешней средой.

Ключевые слова: алгоритмы, модель, дельфи.

Существует довольно много задач гидро и газо-динамики, механики, нанотехнологии и других областей - требующих построения трехмерной модели и исследования поведения отдельных ее элементов или частиц, во взаимодействии и динамическом изменении во времени. К примеру Премия Гордона Белла 2013 года, вручаемая ежегодно за достижения в области высокопроизводительных вычислений, досталась группе исследователей, которые смоделировали возникновение и эволюцию пузырьков жидкости при кавитации (Рис.1). Группа специалистов из Швейцарского федерального технологического института, исследовательского центра IBM, Технического университета Мюнхена и Ливерморской национальной лаборатории в США получила приз в 10 тысяч долларов за свои работы о моделированию кавитации. Ученым удалось получить наиболее подробную модель, которая рассматривает поведение 15 тысяч пузырьков в жидкости [1].

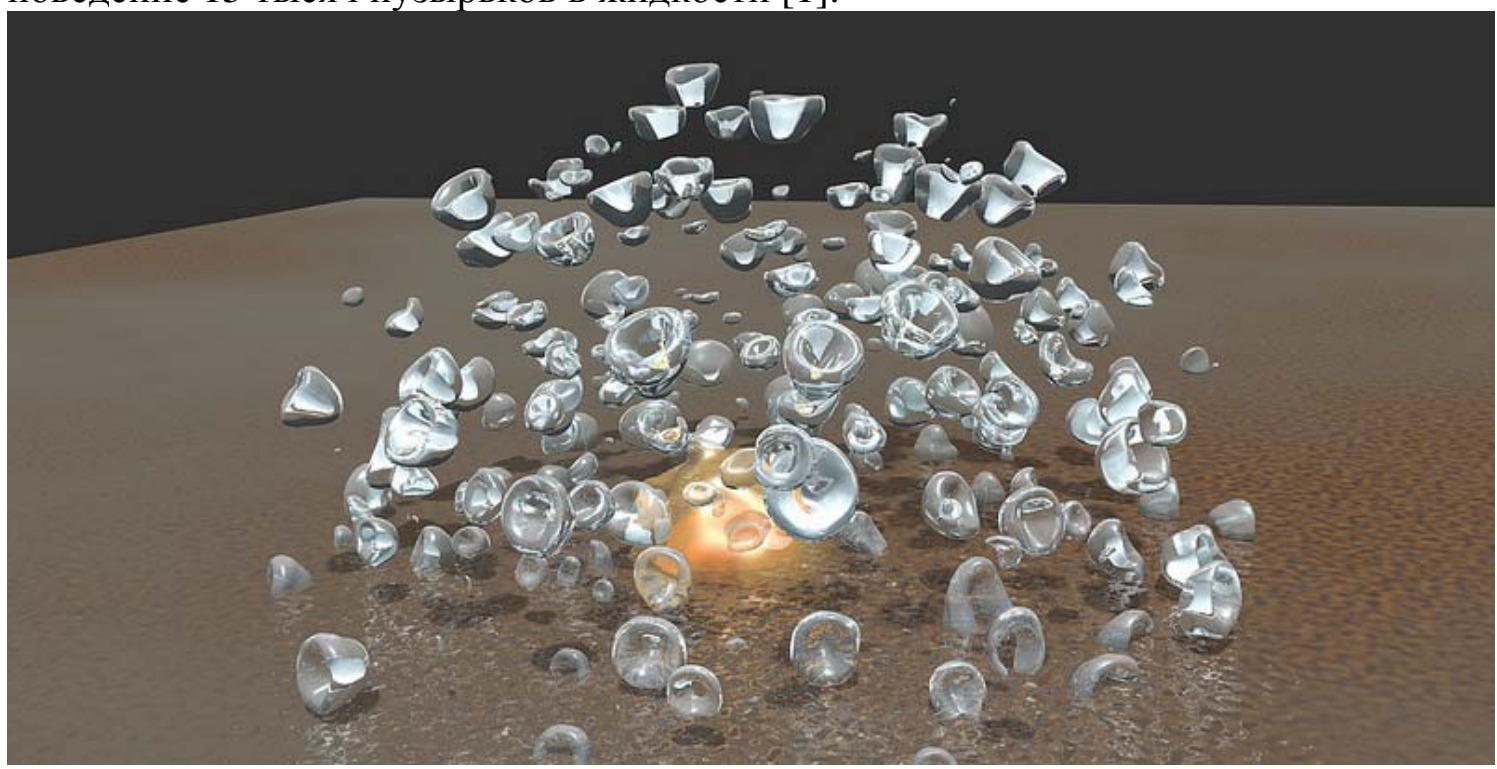

Рисунок 1 - Визуализация исследования кавитационных пузырьков[2]. 
Рассмотрим основы построения подобной модели и алгоритмы на Delphi для еe практической реализации. Введем некоторые упрощения:

- будем рассматривать частицы (атомы) как центральное ядро диаметром 0,1анг. и внешний диаметр (самого атома, на котором начинается взаимодействие с окружающими частицами) порядка 1 анг.

- зададим ограничения внешнего объема - 10 анг. ${ }^{3}$

- количество от 1 до 1000 частиц.

- зададим массив с динамическими данными, для каждого из них, пространственную координату, единичный вектор скорости и уровень кинетической энергии в пределах [0...1].

- введем ограничение подвижности, при 30\% энергии, и менее, частица будет прекращать свое движение.

- зададим законы столкновения частиц, характерные для жидких или газообразных сред.

- а также граничные условия, и начальные уровни энергий.

Создадим приложение и форму (Рис.2.). Построение и отрисовку всех элементов будем производить через DirectX и бибилиотеку OpenGL.

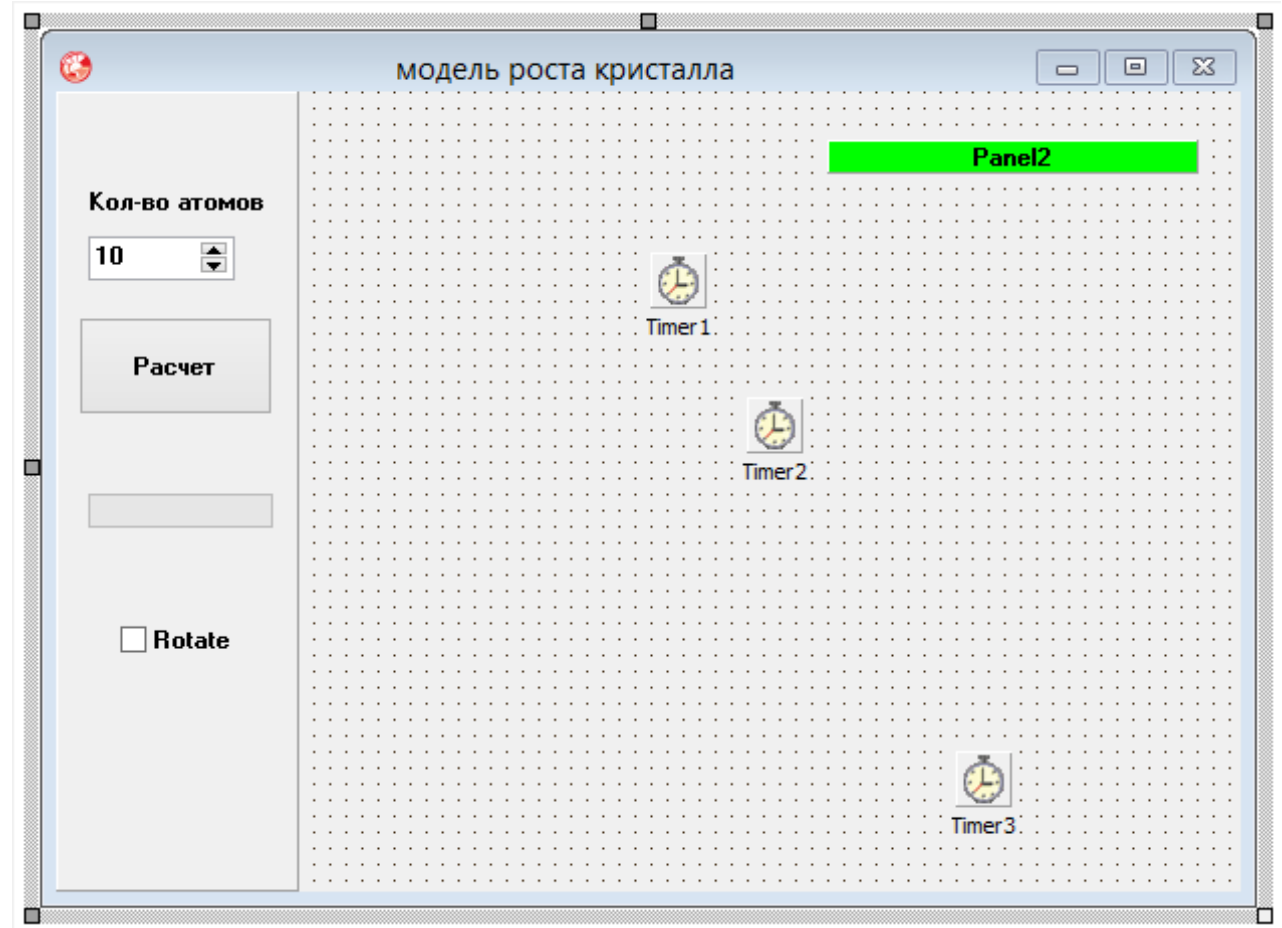

Рисунок 2 - Окно программы.

code Delphi

unit Unit1;

interface

uses

Windows, Messages, SysUtils, Variants, Classes, Graphics, Controls, Forms,

Dialogs, ExtCtrls, OpenGL, ComCtrls, StdCtrls, Spin;

type

TForm1 $=$ class $($ TForm $)$

Timer1: TTimer;

Timer2: TTimer;

Panel1: TPanel; 


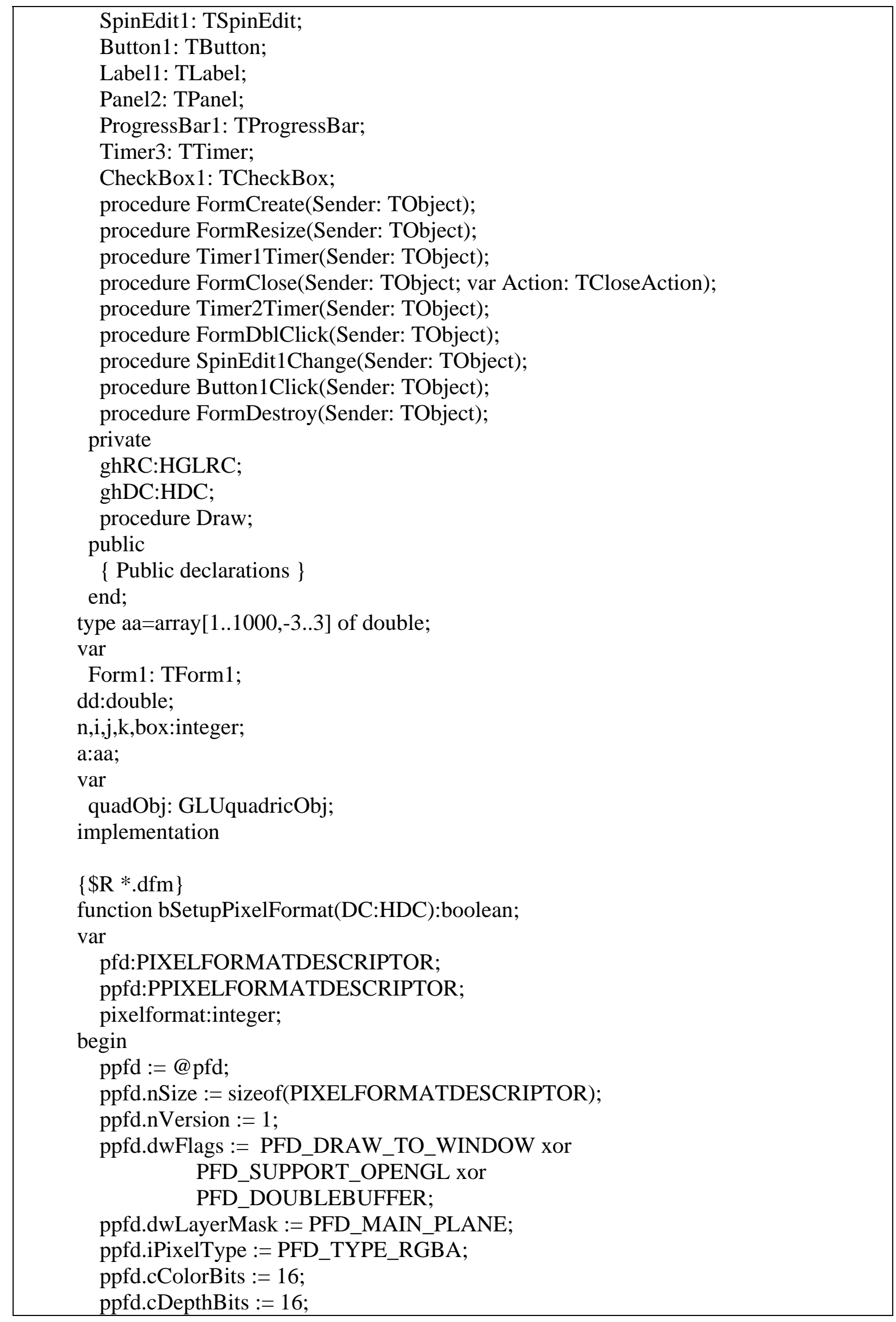




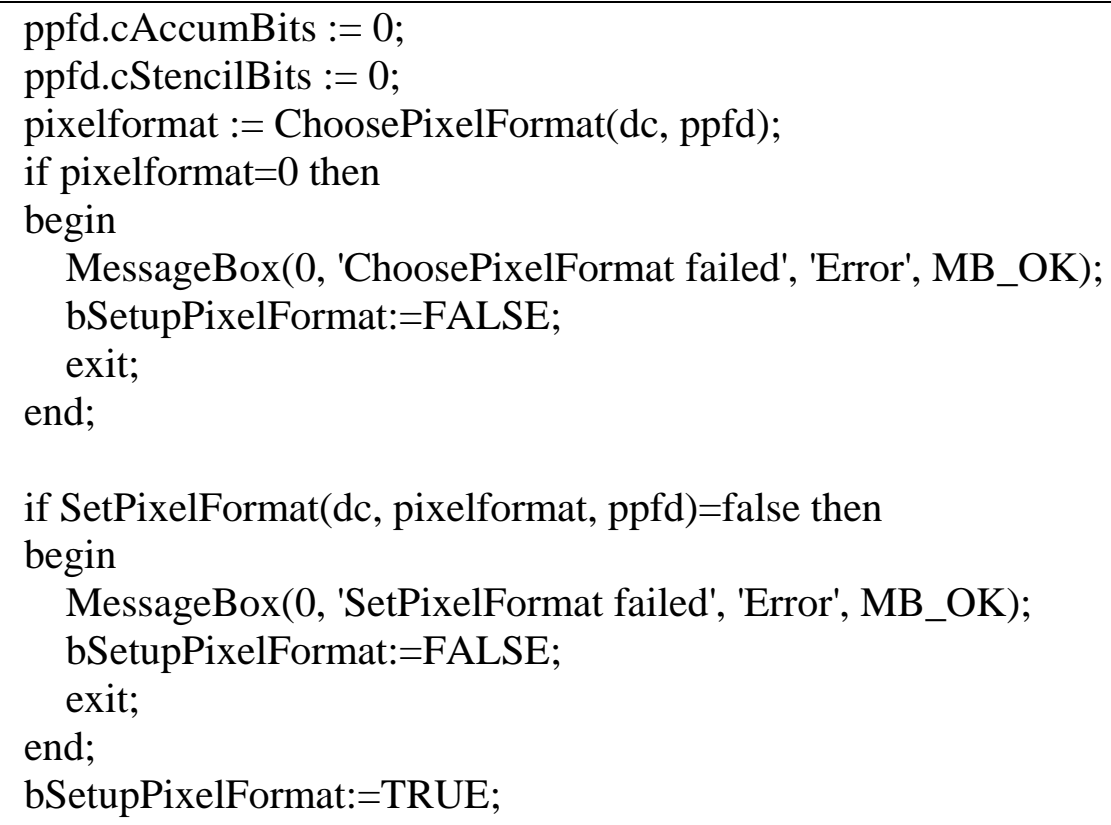




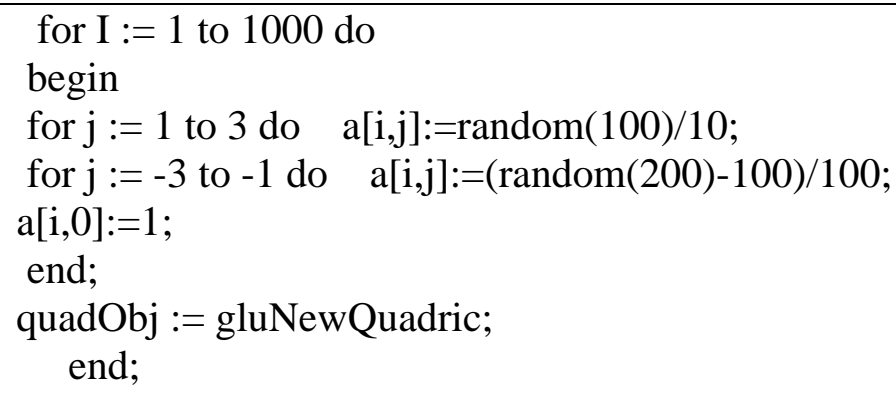


if $\mathrm{a}[\mathrm{i}, 0]>0.3$ then

// движение

$a[i, j]:=a[i, j]+a[i,-j] * a[i, 0]$ else

begin

//алгоритм кристаллизации

end;

if $\mathrm{a}[\mathrm{i}, \mathrm{j}]>$ box then begin $\mathrm{a}[\mathrm{i}, \mathrm{j}] \mathrm{j}$ :=-abs $(\mathrm{a}[\mathrm{i},-\mathrm{j}])$; $\mathrm{a}[\mathrm{i}, \mathrm{j}]$ :=box; end;

if $\mathrm{a}[\mathrm{i}, \mathrm{j}]<0$ then begin $\mathrm{a}[\mathrm{i},-\mathrm{j}]:=\operatorname{abs}(\mathrm{a}[\mathrm{i}, \mathrm{j}]) ; \mathrm{a}[\mathrm{i}, \mathrm{j}]:=0$ end;

end;

if $\mathrm{a}[\mathrm{i}, 1]=0$ then $\mathrm{a}[\mathrm{i}, 0]:=0.9 * \operatorname{abs}(\mathrm{a}[\mathrm{i}, 0])$;

for $\mathrm{j}:=1$ to $\mathrm{n}$ do if $\mathrm{i}<>\mathrm{j}$ then

begin

$\mathrm{s}:=\operatorname{sqrt}(\operatorname{sqr}(\mathrm{a}[\mathrm{i}, 1]-\mathrm{a}[\mathrm{j}, 1])+\operatorname{sqr}(\mathrm{a}[\mathrm{i}, 2]-\mathrm{a}[\mathrm{j}, 2])+\operatorname{sqr}(\mathrm{a}[\mathrm{i}, 3]-\mathrm{a}[\mathrm{j}, 3]))$;

if $s<2$ then

begin

$\mathrm{s}:=(\mathrm{a}[\mathrm{i}, 0]+\mathrm{a}[\mathrm{j}, 0]) / 2$;

$\mathrm{a}[\mathrm{i}, 0]:=\mathrm{s}$;

$\mathrm{a}[\mathrm{j}, 0]:=\mathrm{s}$;

for $\mathrm{k}:=-3$ to -1 do

begin

$\mathrm{s}:=\mathrm{a}[\mathrm{i}, \mathrm{k}]$;

$\mathrm{a}[\mathrm{i}, \mathrm{k}]:=\mathrm{a}[\mathrm{j}, \mathrm{k}]$;

$\mathrm{a}[\mathrm{j}, \mathrm{k}]:=\mathrm{s}$;

end;

end; end; end;

glPopMatrix();

glClear(GL_DEPTH_BUFFER_BIT xor GL_COLOR_BUFFER_BIT);

glColor3f(0,0,1);

glPushMatrix();

for $\mathrm{I}:=1$ to $\mathrm{n}$ do

begin

if $\mathrm{a}[\mathrm{i}, 0]>0.3$ then glColor3f(a[i,0],0,0) else

glColor3f(a[i,0],0,1-a[i,0]);

glPushMatrix();

glTranslatef(a[i,1],a[i,2],a[i,3]); //смещение центра

gluSphere(quadObj, 0.1, 10,10);

glPopMatrix();

end;

glPopMatrix();

glBegin(GL_LINE_STRIP);

glcolor3f $(50,0,0)$;

glVertex3f(50, 0,0);

glVertex3f(0, 0,0$)$;

glcolor3f $(0,10,0)$; 
glVertex3f(0, 50,0);

glVertex3f(0, 0,0);

glcolor3f $(0,0,10)$;

glVertex $(0,0,50)$;

glEnd;

if checkbox1.checked then glRotatef(-1, $\quad 0,0,1)$;

SwapBuffers(ghDC);

end;

procedure TForm1.Timer1Timer(Sender: TObject);

begin

draw;

end;

procedure TForm1.Timer2Timer(Sender: TObject);

begin

timer2.Enabled:=false;

form1.WindowState:=wsmaximized;

end;

procedure TForm1.FormClose(Sender: TObject; var Action: TCloseAction);

begin

if $\mathrm{ghRC}<>0$ then

begin

wglMakeCurrent(ghDC,0);

wglDeleteContext(ghRC);

end;

if $\mathrm{ghDC}<>0$ then

ReleaseDC(Handle, ghDC);

end;

end.

В результате получим следующую визуализацию, без наложения текстур на триангулированные поверхности частиц (Рис.3). Модель динамическая и управляется несколькими таймерами, определяющими временной шаг расчета взаимодействий и координат. Модель делает расчет в реальном времени, и при увеличении числа частиц, более 100, компьютер (оценка производительности 5,7) начинает притормаживать. Как следствие более целесообразным будет разбиение задачи на: выполнение всех расчетов без прорисовки, и отдельным алгоритмом прорисовка элементов в реальном времени. 


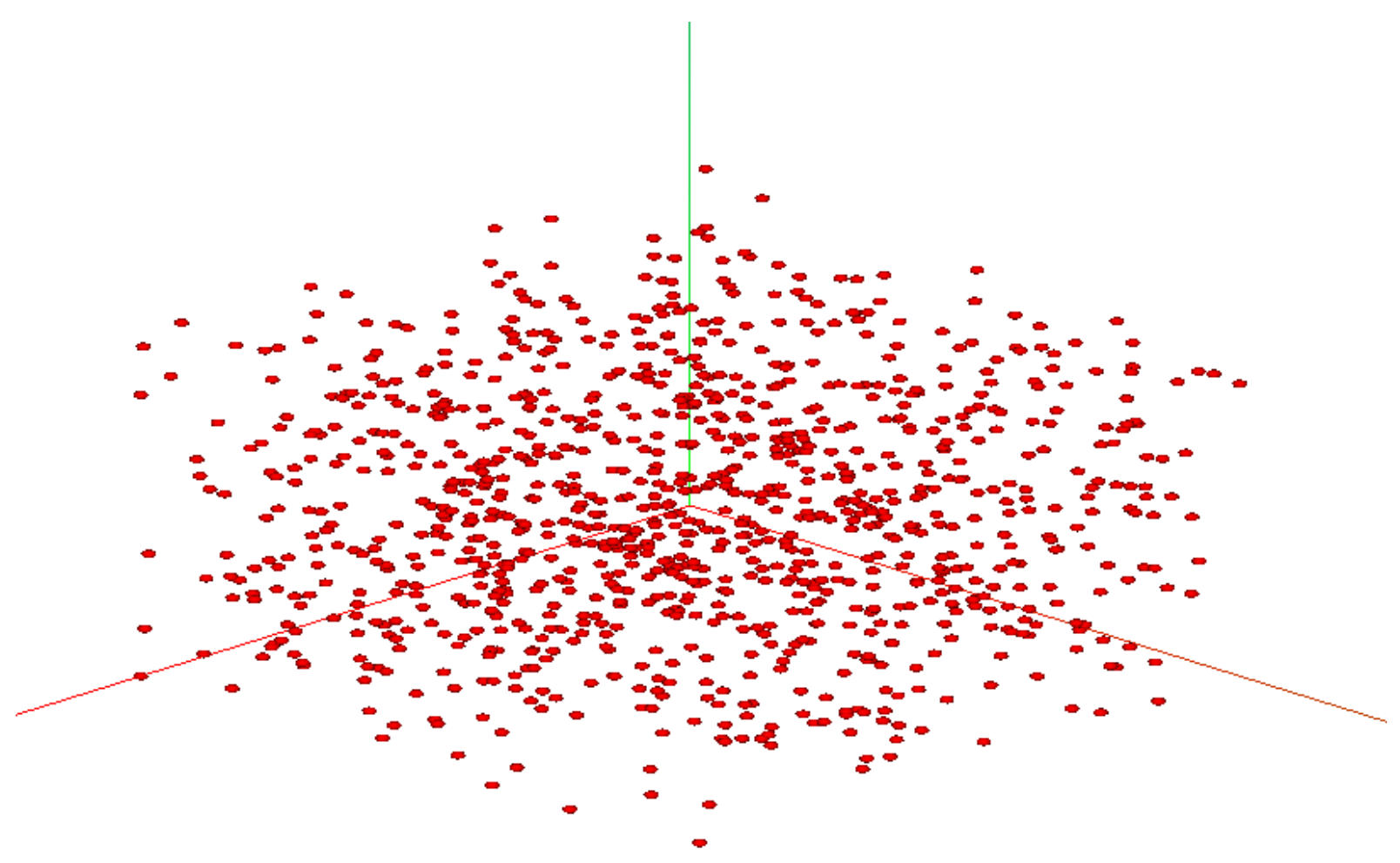

Рисунок 3 - Модель в движении.

\section{Литература}

1. Премию Гордона Белла дали за моделирование пузырьков. [Электронный ресурс]. URL: http://lenta.ru/news/2013/11/25/bellsprize/ (дата обращения: 29.12.2013).

2. Моделирование кавитационных пузырьков получило премию Гордона Белла. [Электронный pecypc]. URL: http://habrahabr.ru/company/ibm/blog/205800/ (дата обращения: 29.12.2013). 